\title{
Artifact removing from EEG recordings using independent component analysis with high-order statistics
}

\author{
Theodor D. Popescu \\ National Instituite for Research and Development in Informatics \\ 8-10 Averescu Avenue, 011455 Bucharest \\ Romania
}

Received: March 15, 2021. Revised: April 1, 2021. Accepted: April 2, 2021. Published: April 8, 2021.

\begin{abstract}
Many methods have been proposed to remove artifacts from EEG recordings especially those arising from eye movements and blinks. Often regression in time and frequency domain on parallel EEG and electrooculographic recordings is used, but this approach can become problematic in some cases. Use of Principal Component Analysis (PCA) has been proposed to remove eye artifacts from multichannel EEG. This method is not effective when the activations from cerebral activity and artifacts have comparable amplitudes. In this paper it is presented a generally applicable method for removing a wide variety of artifacts from EEG recordings based on Independent Component Analysis (ICA) with highorder statistics. The method is applied with good results in the analysis of a sample lowpass event -related potentials (ERP) data.
\end{abstract}

Keywords- Artifacts removing, blind source separation, EEG analysis, independent component analysis, high-order statistics.

\section{INTRODUCTION}

$\mathbf{T}$ HE eye movements, eye blinks, muscle noise, heart signals, and line noise often produce large and distracting artifacts in electroencephalographic (EEG) recordings. Rejecting EEG segments with artifacts larger then an arbitrarily preset value is the most commonly used method for dealing with artifacts removing. When limited data are available, or blinks and muscle movements occur too frequently, the amount of data lost to artifact rejection may be unacceptable.

Several methods have been proposed to remove artifacts from EEG recordings, especially those arising from eye movements and blinks. Often regression in time 1], or frequency domain [2, is performed on parallel EEG and electrooculographic (EOG) recordings to derive parameters for the appearance or spread of EOG artifacts in the EEG channels. Regression methods become problematic when a good regressing channel is not available for each artifact source, as in the case of muscle artifacts. Use of Principal Component Analysis (PCA) has been proposed to remove eye artifacts from multichannel EEG [3. However, PCA cannot completely separate eye artifacts from brain signals, especially when they have comparable amplitudes.

To solve a such problem, there are several software packages and toolboxes that can be used for EEG signal analysis. Examples are the very comprehensive EEGLAB software package [4, LORETA (low-resolution brain electromagnetic tomography) [5], ASA cognitive software [6], and the BIOSIG open-source software tool for biomedical signal processing [7]. Some of the tools evaluate not only EEG but also EMG, for example BrainStorm 8]. A few pattern recognition toolboxes are also available, e.g. PRTools 9]. These tools are in most cases very narrowly specialized, and each has its limitations.

Here we present a generally applicable method for removing a wide variety of artifacts from EEG records based on Independent Component Analysis (ICA), suggested by Makeig et al [10], and starting from a neural network learning algorithm 11. They showed that the ICA algorithms can be used to separate neural activity from muscle and blink artifacts in spontaneous EEG data and reported its use for finding components of EEG and event-related potentials (ERP).

ICA method applied in this paper is based on the assumptions that the signals recorded on the scalp are mixtures of time courses of temporally independent cerebral and artifactual sources, that the potential arising from different parts of the brain, scalp and body are summed linearly at the electrodes, and that propagation delays are negligible. The method uses spatial filters derived by ICA algorithms, and does not require reference channels for each artifact source. Once the independent time courses of different brain and artifact sources are extracted from the data, "corrected" EEG signals can be derived by eliminating the contributions of artifactual sources.

The outline of this paper is as follows. In Section 2 , the proposed approach is presented, including problem formulation and artifact removing from EEG data, 
resulting the artifact-" corrected" EEG recordings. Also, we give the description of the main tool used for artifacts removing from EEG signals, ICA, including models and algorithms used. Section 3 presents a case study having as subject analysis of EEG recordings collected from 13 scalp and 1 EOG electrodes, applied to sample lowpass ERP data for 2 epochs, a data set from literature.

\section{Artifact REMOVIng From EEG RECORDINGS USING ICA}

\section{A. Preliminary}

Independent Component Analysis represents the main tool in Blind Source Separation (BSS) [12, with application to signal analysis in many application areas. It offers, also, a promising area of applications for electroencephalograms (EEG) and magnetoencephalograms (MEG), which are recordings of electric and magnetic fields of signals emerging from neural currents within the brain.

The EEG data represent a matrix, $\mathbf{X}$, containing the EEG signals recorded at different electrodes placed on the scalp. The rows of this matrix are EEG signals and the columns are measurements recorded al different time points. The EEG activity is contaminated by eye movements, blinks, muscle, heart and line noise, which represent a problem for interpretation and analysis of these signals. The main approaches used to remove these artifacts consisted of [13]:

- Rejecting contaminated EEG epochs from the signals, having as effect loss of information.

- Regression in time of frequency domain on EEG and electrooculographic (EOG) recordings simultaneously. This could have as effect subtracting a portion of relevant EEG signal from each recording.

- Due to many noise sources, have no clear reference channels, the approach based on regression methods cannot be used.

The approach proposed in [14, [15] consists to apply ICA to multichannel EEG signals and remove the complex artifacts from recorded original EEG signals. It is proved that this approach is able to detect, separate and remove activity induced in EEG signals from a large variety of artifact sources.

The application of ICA to EEG signals analysis assumes that several conditions are verified, at least approximately: the existence of statistically independent source signals, their instantaneous linear mixing at the sensors, and the stationarity of the mixing and the independent components (ICs).

The independence criterion considers solely the statistical relations between the amplitude distributions of the signals involved, and not the morphology or physiology of neural structures. Thus its validity depends on the experimental situation, and cannot be considered in general.

Because most of the energy in EEG signals lies below $1 \mathrm{kHz}$, each time instance can be considered separately and the propagation of the signals is immediate, there is no need for introducing any time-delay, and the instantaneous mixing is valid.

When considering the underlying source signals as stochastic processes, the requirement of stationarity is in theory necessary to guaranty the existence of a representative distribution of the ICs. In implementation of batch ICA algorithms, the data are considered as random variables, and their distributions are estimated from the whole data set. Thus, the nonstationarity of the signals is not really a violation of the assumptions of the model, but the stationarity of the mixing matrix is crucial [12].

Therefore, ICA-based artifact correction is able to separate and remove a wide variety of artifacts from EEG data by linear decomposition. To conclude, it is based on the following assumptions, on the EEG signals, recorded on the scalp:

- The EEG signals are spatially stable mixtures of the activities of independent cerebral and artifact sources

- The sum of potentials, at the electrodes, coming from different parts of the brain, scalp, and body is linear

- Propagation delays from the sources to the electrodes are insignificant

The last two assumptions are quite reasonable in the case of EEG or MEG data. For enough input data, also the first assumption is reasonable. The presented approach, makes use of spatial filters, provided by ICA algorithms, and does not need a reference channel for each artifact source. After the independent time courses of different brain and artifact sources are estimated, they are extracted from the original EEG data, resulting the artifact-corrected EEG signals, to be used for further investigations.

\section{B. Signal model for $I C A$}

ICA is closely related to the method BSS, or blind signal separation [12]. A "source" means here an original signal, i.e. independent component. "Blind" means that we have very little, if anything, information on the mixing matrix, and make little assumptions on the source signals. ICA is one method, perhaps the most widely used, for performing blind source separation. BSS deals with the problem of recovering multiple independent sources from their mixtures. ICA is one method, perhaps the most widely used, for performing blind source separation. The simple model for BSS assumes the existence of $n$ independent signals $s_{1}(t), \ldots, s_{n}(t)$ and the observation of as many mixtures $x_{1}(t), \ldots, x_{n}(t)$, these mixtures being linear and instantaneous, i.e.

$$
x_{i}(t)=\sum_{j=1}^{n} a_{i j} s_{j}(t)+n_{i}(t)
$$

for each $i=1, n$. This is compactly represented by the mixing equation 


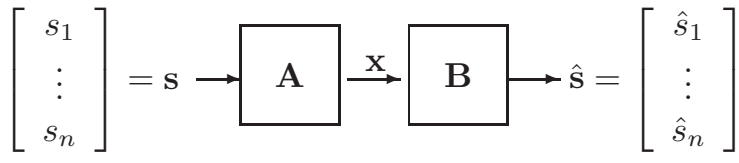

Fig. 1: Mixing and separating. Unobserved signals: s; observations: $\mathbf{x}$; estimated source signals: $\hat{\mathbf{s}}$

$$
\mathbf{x}(t)=\mathbf{A} \mathbf{s}(t)+\mathbf{n}(t)
$$

where $\mathbf{s}(t)=\left[s_{1}(t), \ldots, s_{n}(t)\right]^{T}$ is an $n \times 1$ column vector collecting the source signals, while vector $\mathbf{x}(t)$ collects the $n$ observed signals and the square $n \times n$ "mixing matrix" A contains the mixture coefficients.

In the case of convoluted mixtures [16], the model has the following form:

$$
x_{i}(t)=\sum_{j=1}^{n} \sum_{\tau=0}^{P} a_{i j \tau} s_{j}(t-\tau)+n_{i}(t)
$$

for each $i=1, n$, or compactly

$$
\mathbf{x}(t)=\sum_{\tau=0}^{P} \mathbf{A}(\tau) \mathbf{s}(t-\tau)+\mathbf{n}(t)
$$

The BSS consists in recovering the source vector $\mathbf{s}(t)$ using only the observed data $\mathbf{x}(t)$, the assumption of independence between the entries of the input vector $\mathbf{s}(t)$ and possible some a priori information about the probability distribution of the inputs. It can be formulated as the computation of an $n \times n$ "separating matrix" $\mathbf{W}$ whose output $\hat{\mathbf{s}}(t)$ is an estimate of the vector $\mathbf{s}(t)$ of the source signals, and has the form:

$$
\hat{\mathbf{s}}(t)=\mathbf{W} \mathbf{x}(t)
$$

in the case of an instantaneous mixture and

$$
\hat{\mathbf{s}}(t)=\sum_{\tau=0}^{Q} \mathbf{W}(\tau) \mathbf{x}(t-\tau)
$$

in the case of a convolved mixture.

As we mentioned above, the problem of convoluted mixture of the sources do not rise in the case of EEG signals analysis.

\section{Identificability of the ICA model}

The identificability of the noise-free ICA model has been treated in [17. By imposing the following fundamental restrictions (in addition to the basic assumption of statistical independence), the identifiability of the model can be assured:

1) All the independent components $s_{i}$ with the possible exception of one component, must be non-Gaussian.

2) The number of the observed linear mixtures $m$ must be at least as large as the number of independent components $n$, i.e. $m \geq n$.
3) The matrix A must be of full column rank.

For some algorithm classes these assumptions are not necessary. Usually, it is also assumed that $\mathbf{x}$ and $\mathbf{s}$ are centered. If $\mathbf{x}$ and $\mathbf{s}$ are interpreted as stochastic processes instead of simply random variables, additional restrictions are necessary. At the minimum, one has to assume that the stochastic processes are stationary in the strict sense. Some restriction of ergodicity with respect to the quantities estimated are also necessary.

In the ICA model of eq. (2), it is easy to see that the following ambiguities will hold:

1) We cannot determine the variances (energies) of the independent components. The reason is that, both $\mathbf{s}$ and $\mathbf{A}$ being unknown, any scalar multiplier in one of the sources $s_{i}$ could always be cancelled by dividing the corresponding column $\mathbf{a}_{i}$ in $\mathbf{A}$ by the same scalar. As a consequence we may quite as well fix the magnitudes of the independent components; as they are random variables, the most natural way to do this is to assume that each has unit variance: $E\left[s_{i}^{2}\right]=1$. Then the matrix $\mathbf{A}$ will be adapted in the ICA solution methods to take into account this restriction.

2) We cannot determine the order of the independent components. The reason is that, again both $\mathbf{s}$ and A being unknown, we can freely change the order of the terms in the sum (1), and call any of the independent components the first one.

\section{Algorithms for ICA}

Though many papers purport to introduce "new" methods of solution, the existing framework (and solutions) for blind source separation are often the same [18]. The problem of blind source separation is reduced to a mathematical optimization problem, for which a multitude of techniques are reported. The main differences rest on the varieties of cost functions utilized, based on the kurtosis, mutual information, cross power-spectra, neg-entropy and log-likelihood. In many cases these approaches are the result of different formalisms, and can be shown to be mathematically equivalent 12 .

When the signals are temporal coherent, it is possible to solve BSS problem using only the second-order statistics. If the signals are temporal white or have identical normalized spectral densities, without any information on a priori source distributions, the solution will need higher-order statistics. If the source signal distributions are known, the problem could be solved by maximum likelihood method.

When the BSS problem is solved using only the second-order statistics, it is obtained an algorithm sobi that estimates the original sources based on autocorrelations with several time lags and joint approximate diagonalization [19]. This technique has the virtue that it is usually fairly easy to apply and leads, in many cases, to linear solutions that are simple to compute using standard numerical techniques. On the other hand, 
one can claim that techniques based on second-order statistics are optimal for Gaussian signals only. This is because they neglect the extra information contained in higher-order statistics, which is needed in describing non-Gaussian data. The methods, using higher-order information, allow independent components to have identical distribution.

Concerning the second class of algorithms, based on high-order statistics, with possible application in EEG artifact removing, it can be mentioned: runica algorithm, [20], jader algorithm, 21] and fastica algorithm, 22. All these algorithms are implemented in EEGLB toolbox [4.

In general, the physiological significance of any differences in the results of different algorithms (or different parameter choices in the various algorithms) have not been tested. Applied to simulated, relatively low dimensional data sets for which all the assumptions of ICA are exactly fulfilled, all these algorithms return nearequivalent components.

The runica algorithm, that blindly separates mixtures of independent sources using infomax principle, gives stable decompositions with up to hundred of channels. The jader algorithm uses 4th-order moments (whereas infomax uses implicitly a combination of higher-order moments), but the storage required for all 4th-order moments become impractical for datasets with more than approximate 50 channels. The fastica algorithm, based on a fixed-point technique, quickly computes individual components (one by one). However, the order of the components it finds cannot be known in advance, and performing a complete decomposition is not necessarily faster than infomax. It seems that it may be less stable than infomax for high-dimensional data sets 4.

The component order returned by runica and jader is in decreasing order of the EEG variance accounted for each component. In other words, the lower the order of the component, the more data (neural and/or artifactual) it accounts for. In some cases ICA decompositions with runica and fastica, when run twice on the same data, will differ slightly, because ICA decomposition starts with a random weight matrix, so the convergence is slightly different every time.

Concerning the performance measurement in source separation, several measures of distortion are given in 23], which take into account interference from the other sources as well as noise and algorithmic artifacts, and define the performance criteria that measure separately these contributions.

Beyond the variety of the proposed approaches, it appears that the key for their common success resides in the proper design of the statistical criteria according to which separation is forced.

\section{E. ICA applied to EEG signals}

The ICA algorithms are highly effective at performing source separation in domains where the following assumptions are fulfilled:
1) The mixing medium is linear and propagation delays are negligible.

2) The time courses of the sources are independent.

3) The number of sources is the same as the number of sensors; that is, if there are $n$ sensors, the ICA algorithms can separate $n$ sources.

In the case of EEG signals, we assume that the multichannel EEG recordings are mixtures of underlying brain and artifactual signals. Because volume conduction is thought to be linear and instantaneous, assumption (1) is satisfied. Assumption (2) is also reasonable because the sources of eye and muscle activity, line noise, and cardiac signals are not generally time locked to the sources of EEG activity. Assumption (3) is questionable, because we do not know the effective number of statistically independent signals contributing to the EEG activity.

For EEG analysis, the rows of the input $\mathbf{x}(t)$ are the EEG signals recorded at different electrodes, the rows of the output data $\hat{\mathbf{s}}(t)=\mathbf{B} \mathbf{x}(t)$ are the time courses of activation of the ICA, and the columns of the inverse matrix $\mathbf{B}^{-1}$ give the proiection strengths of the respective components onto the scalp sensors. The scalp topographies of the components provide information about the location of the sources. "Corrected" EEG signals can then be derived as $\mathbf{x}^{\prime}(t)=\mathbf{B}^{-1} \hat{\mathbf{s}}^{\prime}(t)$, where $\hat{\mathbf{s}}^{\prime}(t)$ is the matrix of activation waveforms, $\hat{\mathbf{s}}(t)$, with rows representing artifactual components set to zero.

The ICA algorithms are related to PCA. Singular value decomposition, 24] is used to derive the principal component of EEG signals. Multichannel EEG recordings can be expressed by a $m$ (time points) $\times n$ (channels) matrix, E, and descomposed as a product of three matrices, $\mathbf{E}=\mathbf{U S V} \mathbf{V}^{T}$, where $\mathbf{U}$ is an $m \times n$ matrix such that $\mathbf{U}^{T} \mathbf{U}=\mathbf{I}, \mathbf{S}$ is an $n \times n$ diagonal matrix, and $\mathbf{V}$ is an $n \times n$ matrix such that $\mathbf{V}^{T} \mathbf{V}=\mathbf{V} \mathbf{V}^{T}=\mathbf{I}$. If $\mathbf{E}$ is an EEG epoch of $n$ channels and $m$ time points, $\mathbf{U}$ contains its $n$ normalized principal component waveforms that are decorrelated linearly and can be remixed to reconstruct the original EEG. $\mathbf{S}$ contains the $n$ amplitudes of the $n$ principal components waveforms. The eigenvector matrix, $\mathbf{V}$, is essentially a set of topographic scalp maps, similar to the columns of the $\mathbf{B}^{-1}$ matrix found by ICA.

PCA finds orthogonal directions of greatest variance in the data, whereas ICA component maps may be nonorthogonal. In general, there is no reason why neurobiologically distinct EEG sources should be spatially orthogonal to one another. Therefore, PCA should not in general effectively segregate each EEG source such as brain, cardiac, and eye movement generators, into a separate component.

\section{EXPERIMENTAL RESULTS}

The data used in this case study represent an EEG time series collected from 13 scalp and 1 EOG electrodes. The ICA analysis is applied to sample lowpass ERP data for 2 epochs and 312 frames per data epoch, at $312.5 \mathrm{~Hz}$ sampling rate. The data are used in many case studies (see 4], among others). 
The original EEG data for 2 conditions, are shown in Fig. 2 and Fig. 3 for the channels 1-7 (Fz, Cz, Pz, Oz, F3, F4, C3) and for the channels 8-14 (C4, T3, T4, P3, $\mathrm{P} 4, \mathrm{Fpz}, \mathrm{EOG})$, respectively.

Fig. 4 and Fig. 5 present the derived ICA component activations, when jader algorithm has been used.

Starting from these results we decided that sources $3,4,5,6,9$ and 11 could represent artifactuals components. After eliminating of these 6 components, by zeroing out the corresponding rows of the activation matrix $\mathbf{s}(t)$ and projecting the remaining components onto the scalp electrodes, the "corrected" EEG data (Fig. 6 and Fig. 7) were free of artifactual components.

To evaluate the effect of artifact removing by independent component analysis, we present in Fig. 8 and Fig. 9 the ERP filtered data with channel locations on the scalp, for 2 epochs, before and after after artifact removing, respectively.

Similar, in Fig. 10 and Fig. 11 are presented the ERP filtered data for all channels, for 2 epochs, before and after after artifact removing, respectively.

From Fig. 5, Fig. 6, Fig. 7 and Fig. 8 can be noted the effect of artifact removing, resulting a reduction in ERP signal amplitude for all channels, as the effect of artifact components removing in "corrected" EEG signals.

\section{COnClusions}

Although the neural mechanisms that generate EEG are not fully known, the assumptions of the ICA algorithms are generally compatible with a widely assumed model where EEG data recorded at multiple scalp sensors are a linear sum at the scalp electrodes of activations, generated by distinct neural and artifactual sources. ICA opens new perspectives into many brain and non-brain phenomena analysis, contained in multichannel EEG records, by separating data into a sum of temporally independent components. ICA appears to be a generally applicable and effective method for removing a wide variety of artifacts from EEG records, because their time courses are generally temporally independent and spatially distinct from sources of cerebral activity. In addition to EEG artifact removal, ICA decomposition can be equally applicable to other types of multichannel biomedical data for which linear summation of the activations can be assumed.

\section{ACKNOWLEDGMENT}

The author thanks the Executive Agency for Higher Education, Research, Development and Innovation Funding (UEFISCDI) and Ministry of Research and Innovation, for the support under Contract 2019-2022 Core Program, Project PN 301, RO-SmartAgeing.

\section{REFERENCES}

[1] G. Gratton, M. G. Coles and E. Donchin, "A new method for off-line removal of ocular artifact", Electroencephalography and Clinical Neurophysiology, vol. 55, pp. 468-484, 1983.
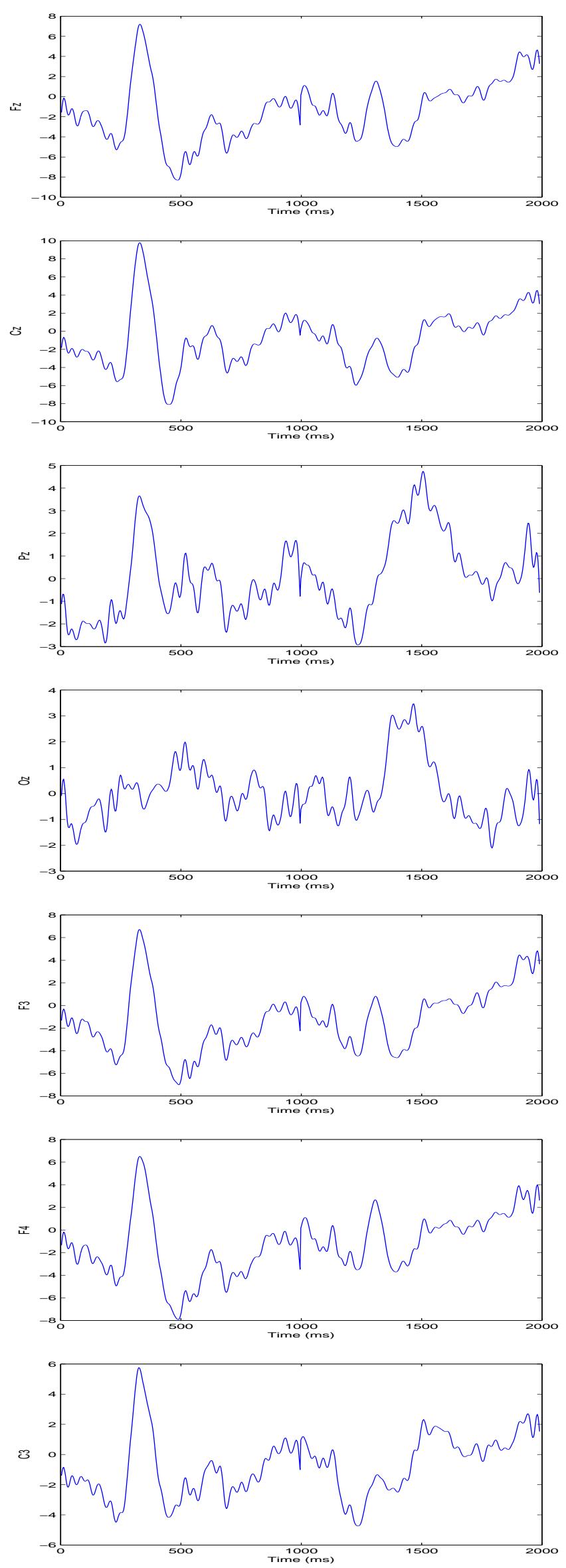

Fig. 2: ERP filtered data for 2 epochs, channels 1-7 

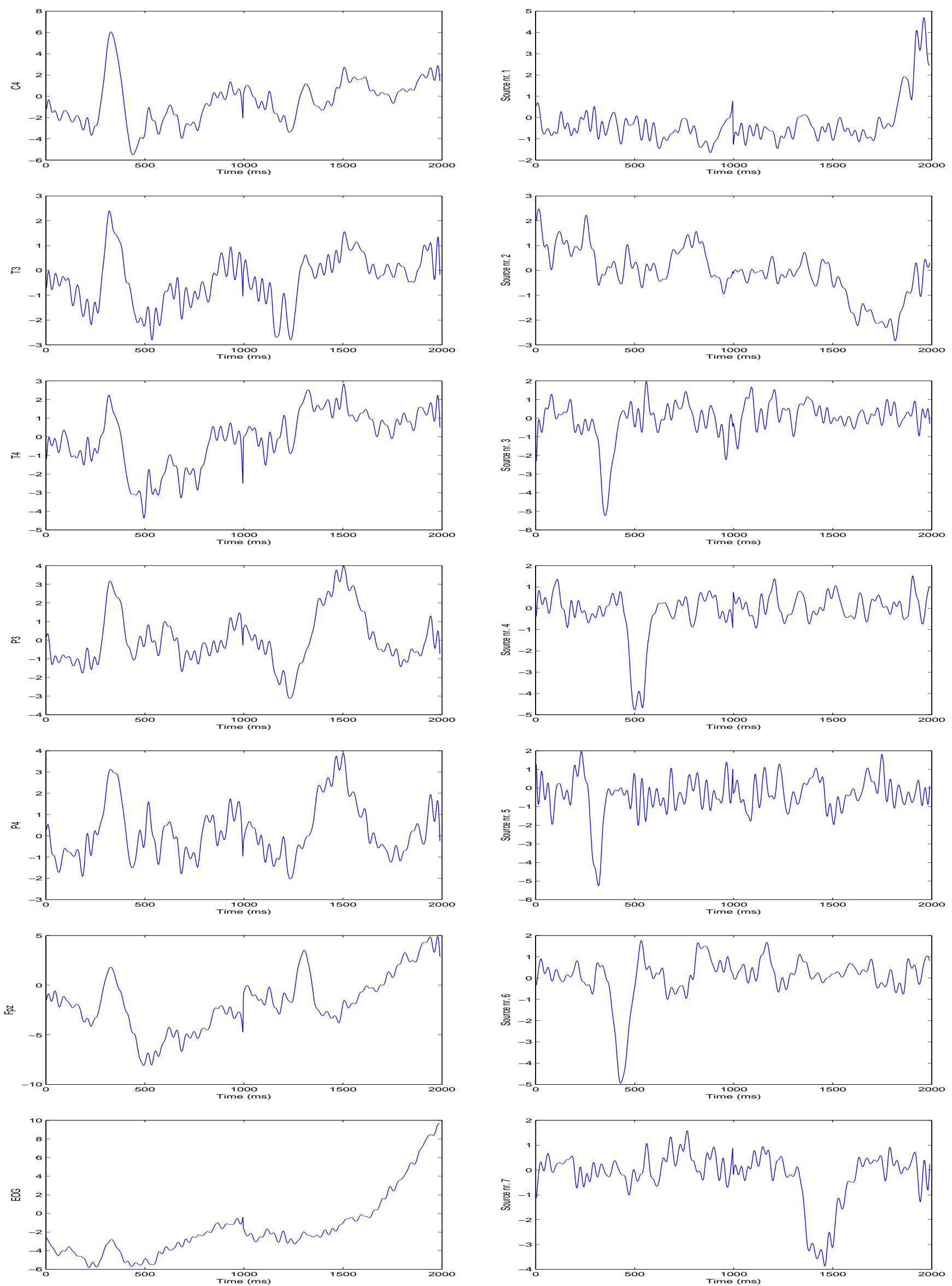

Fig. 3: ERP filtered data for 2 epochs, channels 8-14

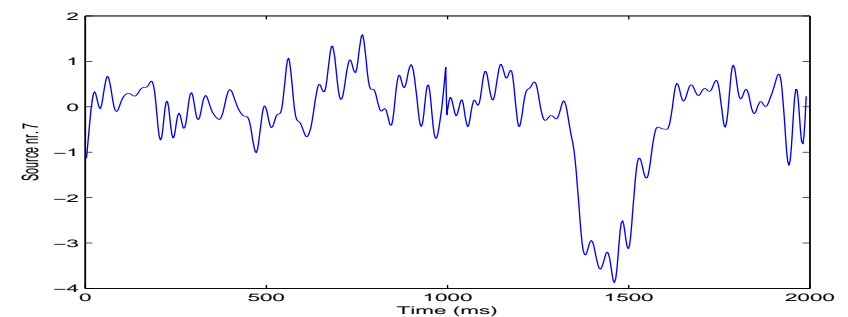

Fig. 4: Estimated sources 1-7 for 2 epochs 

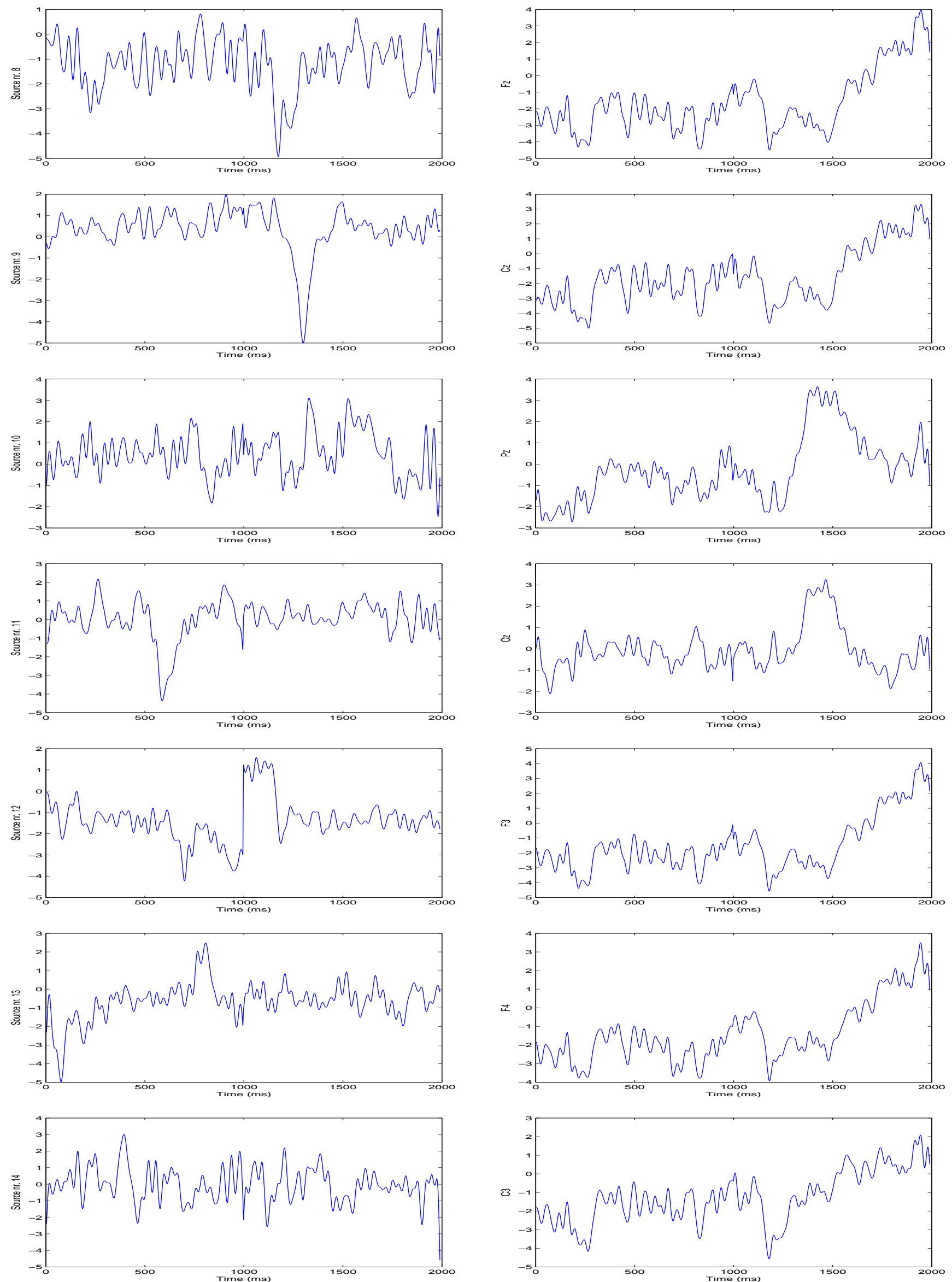

Fig. 5: Estimated sources 8-14 for 2 epochs

Fig. 6: ERP filtered data after artifact removing for 2 epochs, channels 1-7 

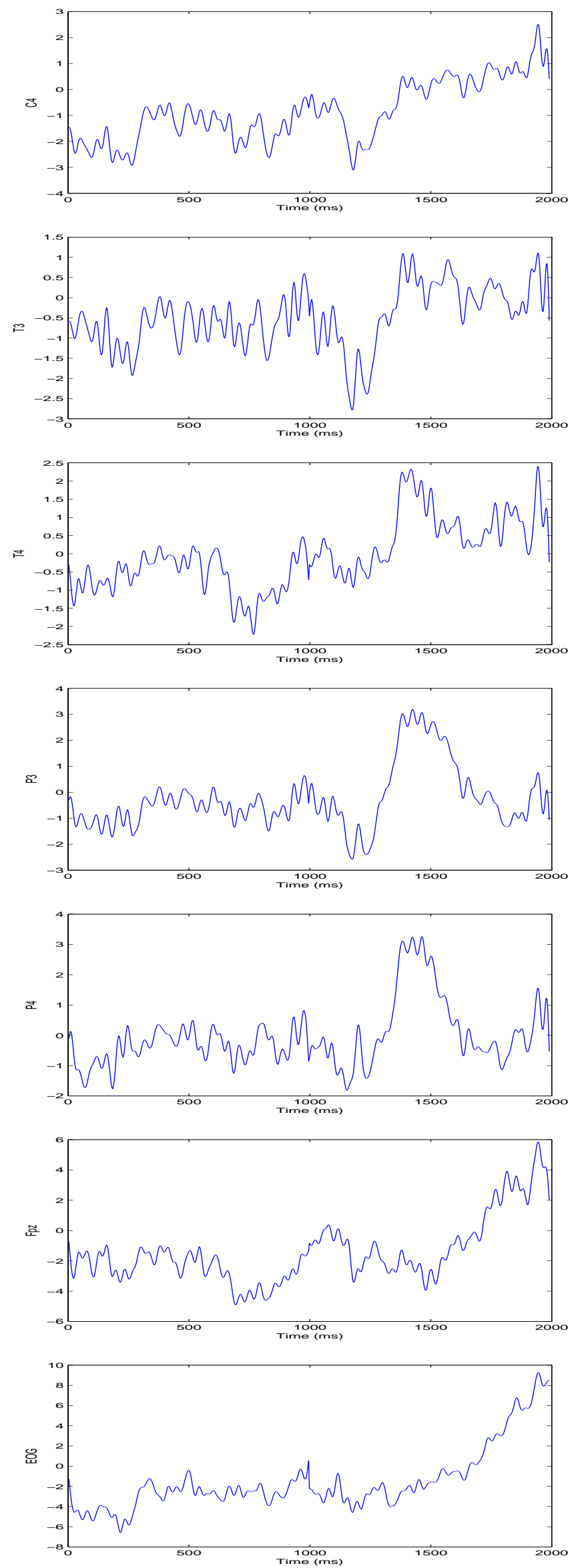

Fig. 7: ERP filtered data after artifact removing for 2 epochs, channels 8-14 


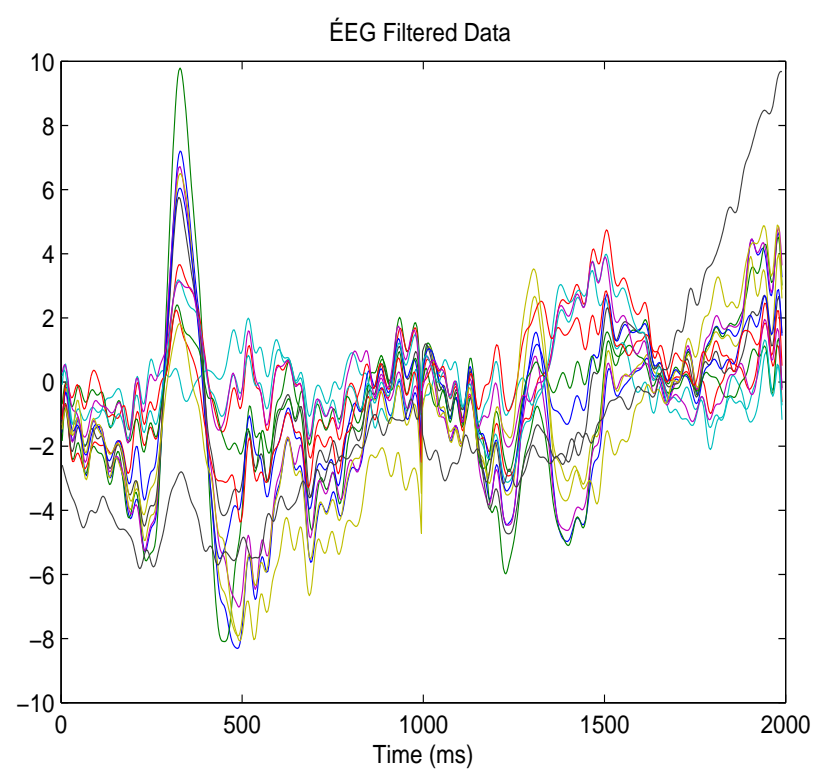

Fig. 10: ERP filtered data for 2 epochs

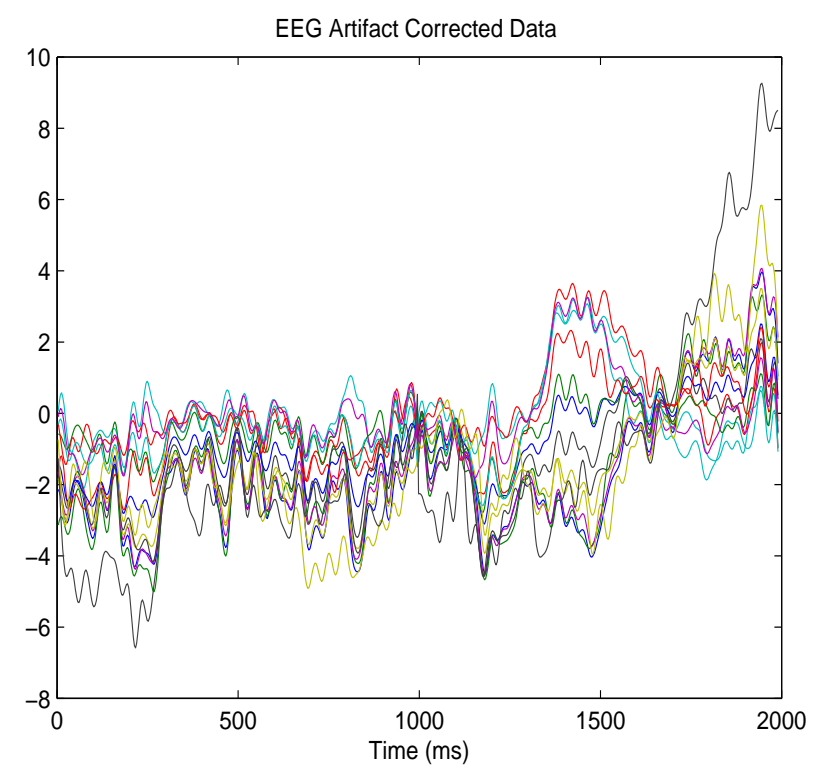

Fig. 11: ERP filtered data for 2 epochs after artifact removing
[2] J. C. Woesternburg, M. N. Verbaten and J. L. Slangen, "The removal of the eye-movement artifacts from the EEG by regression analysis in the frequency domain", Biological Psychology, vol. 16, pp. 127-147, 1983.

[3] P. Berg and M. Sherg, "Dipole models of eye activity and its application in the removal of eye artifacts from the EEG and MEG", Clinical Physics and Physiological Measurements, vol. 12, Supplement A, pp. 49-54, 1991.

[4] A. Delorme and S. Makeig. "EEGLAB: an open source toolbox for analysis of single-trial EEG dynamics including independent component analysis", Journal of Neuroscience Methods, vol. 134, no. 1, pp. 9-21, 2004.

[5] R. D. Pascual-Marqui, M. Esslen, K. Kochi, and D. Lehmann, "Functional imaging with low-resolution brain electromagnetic tomography (LORETA): a review", Methods and findings in experimental and clinical pharmacology, vol. 24, Suppl C:91-95, 2002.

[6] F. Zanow and T. R. Knsche, "ASA - Advanced Source Analysis of continuous and event-related EEG/MEG signals", Brain Topography, vol. 16, no. 4, pp. 287-290, 2004.

[7] A. Schlogl, C. Keinrath, D. Zimmermann, R. Scherer, R. Leeb, and G. Pfurtscheller, "A fully automated correction method of EOG artifacts in EEG recordings", Clinical Neurophysiology, vol. 118, no. 1, pp. 98-104, 2007.

[8] F. Tadel, S. Baillet, J. C. Mosher, and D. Pantazis, "Brainstorm: A user-friendly application for MEG/EEG analysis", Computational Intelligence and Neuroscience, Jan. 2011.

[9] R. P. W. Duin, P. Juszczak, P. Paclik, E. Pekalska, D. de Ridder, D. M. J. Tax, and S. Verzakov, "PRTools 4.1, A Matlab Toolbox for Pattern Recognition", 2007.

[10] S. Makeig, A. J. Bell, T. P. Jung and T. J. Sejnowski, "Independent component analysis of electroencephalographic data", in D. Touretzky, M. Mozer, M. Hasselmo (Eds.), Advances in neural information Processing Systems, vol. 8, pp. 145-151, Cambridge, MA, MIT Press, 1996.

[11] A. J. Bell and T. J. Sejnowski, "An information maximization approach to blind separation and blind deconvolution", Neural Computation, vol. 7, pp. 1129-1159, 1995.

[12] A. Hyvärinen, J. Karhunen and E. Oja, Independent Component Analysis, John Wiley \& Sons, Inc, 2001.

[13] https://cnl.salk.edu/ jung/artifact.html

[14] T. P. Jung, S. Makeig, C. Humphries, T.W. Lee, M. J. McKeown, v. Iragui and T. J. Sejnowski, "Removing electroencephalographic artifacts by blind source separation," Psychophysiology, vol. 37, no. 2, pp. 163-178, 2000.

[15] T. P. Jung, S. Makeig, W. Westerfield, J. Townsend, E. Courchesne and T. J. Sejnowski, "Removal of eye activity artifacts from visual event-related potentials in normal and clinical subjects," Clinical 
Neurophysiology, vol. 111, pp. 1745-1758, 2000.

[16] L. Parra and C. Spence, "Convolutive blind source separation of non-stationary signals", IEEE Trans. on Speech and Audio Processing vol. 8, no. 3, pp. 320-327, 2000.

[17] P. Comon, "Independent component analysis - a new concept ?", Signal Processing, vol. 6, pp. 287$314,1994$.

[18] R. Der, "Blind signal separation", Technical Report, Department of Electrical \& Computer Engineering, McGill University , 2001.

[19] A. Belouchrani, K. Abed Meraim, J. F. Cardoso, and E. Moulines, "A blind source separation technique using second - order statistics", IEEE Trans. Signal Processing, vol. 45, pp. 434-444, 1997.

[20] A. J. Bell and T. J. Sejnowski, "An information maximization approach to blind separation and blind deconvolution", Neural Computation, vol. 7, pp. 1129-1159, 1995.

[21] J. F. Cardoso and A. Souloumiac, "Blind beamforming for non gaussian signals", IEE Proceedings -F, vol. 140, no. 6, pp. 362-370, 1993.

[22] A. Hyvärinen and E. Oja, " A fast fixed-point algorithm for independent component analysis", Neural Computation, no. 9, pp. 1483-1492, 1997.

[23] R. Gribonval, L. Benaroya, E. Vincent and C. Fevotte, "Proposal for performance measurement in source separation", Publication Interne, No. 1501, IRISA, 2002.

[24] G. H. Golub and C. F. V. Loan, Matrix Computation, The John Hopkins University Press, 1989.

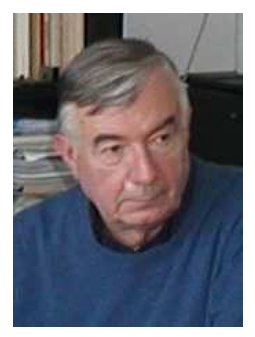

Theodor D. Popescu was born in Rosiori de Vede, Romania, on July 4, 1949. He received his M.Sc. degree and Eng.Sc.D. (PhD) degree, both in Automatic Control, from "Politehnica" University of Bucharest in 1972 and 1983, respectively. He has been a senior research scientist with the National Institute for Research and Development in Informatics, ICI Bucharest, since 1972. His research activities are focused on the fields of system identification and parameter estimation, time series analysis, signal processing, change detection and diagnosis, independent component analysis, blind source separation, time-frequency analysis, biomedical signal analysis. He is author or co-author of more than 150 papers and of five books. He was awarded with "Tudor Tanasescu" Prize of the Romanian Academy in 1995, for his works in the field of detection of changes in dynamics of signals and systems and General Association of Engineers in Romania Prize in 2000 in the field of Informa- tion Technology. He was granted by The Royal Society (1992), DAAD (1992, 1995, 1999), JSPS (1996, 1999), Australian Research Council(2002), CERGE-EI (2002). He held conferences and seminars in the Institute of Systems Science, Beijing, Institute of Technology, Beijing, University of Lancaster, Wuppertal University, Kyushu Institute of Technology, Oita University, Saga University, Tottori University, Kyoto Institute of Technology, CERGE-EI Prague and Spatial Co. Kuwait. He has been a Senior Member of IEEE since 2002.

Sources of funding for research presented in a scientific article or scientific article itself

Executive Agency for Higher Education, Research, Development and Innovation Funding (UEFISCDI) and Ministry of Research and Innovation, for the support under Contract 20192022 Core Program, Project PN 301, ROSmartAgeing

\section{Creative Commons Attribution License 4.0 (Attribution 4.0 International, CC BY 4.0)}

This article is published under the terms of the Creative Commons Attribution License 4.0

https://creativecommons.org/licenses/by/4.0/deed.en_US 